\title{
Dendritic cells in autoimmunity, infections, and cancer
}

\author{
Francisco J. Quintana ${ }^{1,2}$ (1)
}

Received: 20 December 2016 / Accepted: 20 December 2016 / Published online: 16 January 2017

(C) Springer-Verlag Berlin Heidelberg 2017

Dendritic cells (DCs) play a central role in the control of the adaptive immune response. DCs drive the differentiation of effector $\mathrm{T}$ cells (Teffs) that fight off pathogens, but the dysregulated activity of Teffs can result in immunopathology and autoimmune disorders. Consequently, it is not surprising that DCs also promote the development of regulatory $\mathrm{T}$ cells (Tregs) that suppress harmful immune responses. Thus, DCs play a central role in the control of the $\mathrm{T}$ cell response, providing an attractive target for immunotherapy (Fig. 1). This issue of Seminars in Immunopathology focuses on the role of DCs in the control of the immune response in the context of autoimmunity, infections and cancer, and their potential as targets for therapeutic intervention.

Several DC populations have been identified that differ in their ability to prime Teffs and Tregs. Based on the current knowledge acquired during the study of the central nervous system autoimmune disease multiple sclerosis (MS) and its experimental model experimental autoimmune encephalomyelitis, Sie and Korn review the role of DCs in shaping tissue-specific T cell autoimmunity [1]. Moreover, they evaluate the contribution of DCs in the peripheral organs and the target tissue, as

This article is a contribution to the special issue on Dendritic Cell Subsets and Immune-mediated Diseases - Guest Editor: Francisco J. Quintana

Francisco J. Quintana

fquintana@rics.bwh.harvard.edu

1 Ann Romney Center for Neurologic Diseases, Brigham and Women's Hospital, Harvard Medical School, 60 Fenwood Road, Boston, MA 02115, USA

2 The Broad Institute of MIT and Harvard, Cambridge, MA, USA well as their modulation by disease-modifying therapies currently used in MS.

Under certain scenarios, DCs show tolerogenic properties that allow them to limit autoimmune $\mathrm{T}$ cell responses. Takenaka and Quintana review the DC populations that display a tolerogenic phenotype, their development, and stability [2]. In addition, they discuss pathways known to induce tolerogenic DCs, which may offer opportunities for therapeutic intervention. On a related issue, Orgad et al. focus on a newly identified population of tolerogenic DCs characterized by the expression of perforin and Granzyme A [3]. Interestingly, these cells are relevant not only for the control of tissue-specific autoimmunity, but also for the control of inflammatory responses in fat associated to metabolic disorders.

Although DCs with tolerogenic function have been identified, little is known about the molecular mechanisms that control them. Devi and Anandasabapathy review the development of tolerogenic DCs in different tissues and provide an exhaustive evaluation of the transcriptional programs that control them [4]. Finally, one of the best-characterized tolerogenic functions of DCs is the promotion of Treg differentiation. Waisman et al. discuss the complex mechanisms that mediate the control of Tregs by DCs and their therapeutic relevance [5].

Cytokines, pathogen-, and danger-associated molecular patterns (PAMPs, DAMPs) are known to have important effects on DC development and function. However, additional molecules are also involved in the regulation of DCs. For example, DCs express receptors responsive to neurotransmitters released by sympathetic fibers that innervate tissues. Basso and coworkers discuss how neurotransmitters from the sympathetic nervous system modulate DC function [6]. In addition, Minarrieta et al. review 
Fig. 1 Molecular mechanisms controling DC development

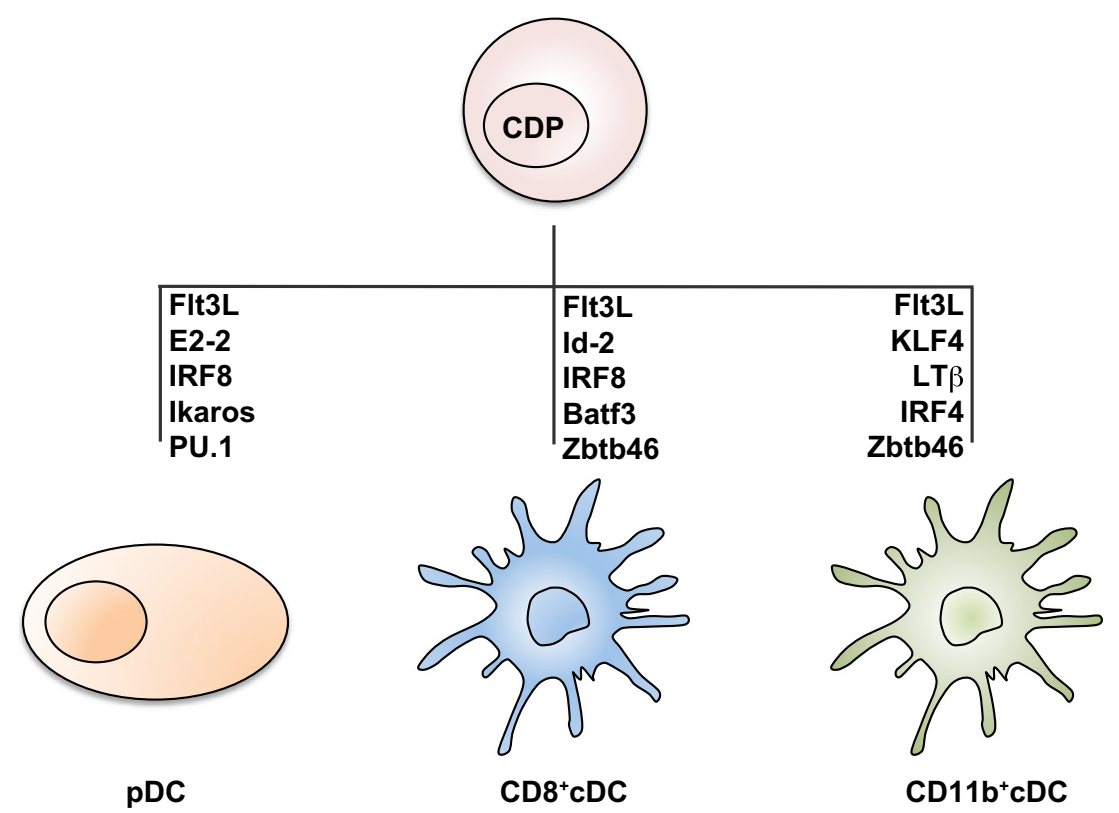

the role of metabolites produced by the host and pathogens in the regulation of DC function [7].

Parasites and bacteria manipulate the immune response to establish chronic infections. One of the strategies used to achieve this goal is to modulate DC function. Motran et al. review the mechanisms involved in the regulation of DC function by parasites [8]. In addition, Marim et al. discuss the mechanisms involved in the control of caspase-1 activation and IL-1 production by Brucella abortus and its role in the establishment of chronic infection and immunopathology [9].

Finally, immune-based therapies have shown promising results for the treatment of cancer. DC vaccines have been studied for the treatment of cancer, indeed some of these vaccines have already been approved for clinical use. Reardon and Mitchell review the potential and limitations of DC vaccines as immunotherapeutic tools for glioblastoma [10].

In conclusion, this issue of Seminars in Immunopathology provides a comprehensive overview on the role of DCs in health and disease, the mechanisms that control them, and potential targets for their therapeutic modulation. We present this issue with the expectation that these reviews will guide new and efficacious immunotherapies.

\section{References}

1. Sie C, Korn T (2017) Dendritic cells in central nervous system autoimmunity. Semin. Immunopathol. doi:10.1007/s00281-0160608-7
2. Takenaka MC, Quintana FJ (2017) Tolerogenic dendritic cells. Semin. Immunopathol. doi:10.1007/s00281-016-0587-8

3. Orgad R, Nathansohn-Levi B, Kagan S, Reisner Y (2017) Novel immuno-regulatory role of perforin positive dendritic cells. Semin. Immunopathol. doi:10.1007/s00281-016-0589-6 [erratum: doi:10.1007/s00281-016-0598-5]

4. Devi KSP, Anandasadapathy N (2017) The origin of DCs and capacity for immunologic tolerance in central and peripheral tissues. Semin. Immunopathol. doi:10.1007/s00281016-0602-0

5. Waisman A, Lukas D, Clausen BE, Yogev N (2017) Dendritic cells as gatekeepers of tolerance. Semin. Immunopathol. doi:10.1007 /s00281-016-0583-Z

6. Takenaka MC, Guereschi MG, Basso AS (2017) Neuroimmune interactions: dendritic cell modulation by the sympathetic nervous system. Semin. Immunopathol. doi:10.1007 /s00281-016-0590-0

7. Minarrieta L, Ghorbani P, Sparwasser T, Berod L (2017) Metabolites: deciphering the molecular language between DCs and 1 their environment. Semin. Immunopathol. doi:10.1007 /s00281-016-0609-6

8. Motran CC, Ambrosio LF, Volpini X, Celias DP, Cervi L (2017) Dendritic cells and parasites: from recognition and activation to immune response instruction. Semin. Immunopathol. doi:10.1007 /s00281-016-0588-7

9. Marim FM, Franco MMC, Gomes MTR, Miraglia MC, Giambratolomei GH, Oliveira SC (2017) The role of NLRP3 and AIM2 in inflammasome activation during Brucella abortus Infection. Semin. Immunopathol. doi:10.1007/s00281-0160581-1

10. Reardon DA, Mitchell DA (2017) The development of dendritic cell vaccine-based immunotherapies for glioblastoma. Semin. Immunopathol. doi:10.1007/s00281-0160616-7 